\title{
Advanced liver disease: It is not all about Covid-19
}

\author{
Del Pino Bellido Pilar ${ }^{1 *}$, Guerra Veloz Maria Fernanda ${ }^{1}$, Muñoz Garcia-Borruel Maria ${ }^{1}$, Salamanca Rivera Elena ${ }^{2}$ and Carmona Soria Isabel $^{1}$ \\ ${ }^{1}$ Gastroenterology and Hepatology Department. Virgen Macarena University Hospital, Seville, Spain \\ ${ }^{2}$ Infectious Diseases Department. Virgen Macarena University Hospital, Seville, Spain
}

\begin{abstract} there is limited data linking chronic liver disease and Covid-19. with COVID-19.

\section{Introduction}

Coronavirus disease 2019 (COVID-19) is caused by severe acute respiratory syndrome coronavirus 2 (SARS-CoV-2), which was discovered in Wuhan, China in December 2019. Older patients and those with pre-existing medical conditions have been identified as populations at risk of a severe disease course. Patients with advanced chronic liver disease may be at increased risk of infection and/or severe course due to the cirrhosis-associated immune dysfunction. However, there is limited data linking chronic liver disease and Covid-19 [1-6].
\end{abstract}

Patients with advanced chronic liver disease may be at increased risk of infection and/or severe course due to the cirrhosis-associated immune dysfunction. However,

Patients with cirrhosis are known to have abnormalities of immune function (immunodeficiency), as well as systemic inflammation, which is the pathophysiological hallmark of increased susceptibility to infection. Patients with cirrhosis are at a high risk of having a severe course of the influenza, including the development of organ failures. However, there is limited data on the course of Covid-19 in patients with chronic liver disease (CLD) and whether SARS-Cov2 can increase the risk of decompensation or development of acute-on-chronic liver failure (ACLF). Actual data indicates a prevalence (0-11\%) of pre-existing liver conditions in patients

We present two cases of patients with decompensated liver disease that were evaluated for the SARS-Cov2 due to the new hospital policy of testing all patients who require hospital admission. One of them was asymptomatic, while the other presented mild respiratory symptoms. We believe that COVID-19 did not worsen liver function in our patients, and as such did not increase their risk of mortality or develop ACLF during admission.

\section{Case report}

An adult patient with HIV-HCV coinfection (both treated) and decompensated cirrhosis Child Pugh B9, with a recent hospital admission for variceal bleeding, radiological ascites, and multicenter hepatocellular carcinoma with portal invasion, was admitted to our emergency unit with hemodynamic instability due to upper gastrointestinal bleeding. Blood tests results showed: hemoglobin $11.8 \mathrm{~g} / \mathrm{dL}$, platelet count $123000 / \mu \mathrm{L}$; international normalized ratio (INR) 1.10 and positive Sars-Cov2 PCR test (Figure 1). Medical therapy with terlipressin, pantoprazole and ceftriaxone was started. Urgent gastroscopy was performed and revealed active bleeding from post-banding ulcer, which was treated successfully with injection of ethoxysclerol. During the admission in the infectious diseases service, he presented new episodes of melena and worsening ascites, medical therapy and blood transfusions were started with good response. Even though the Sars-Cov2 PCR was positive he did not present respiratory symptoms during the admission. Also, the chest radiography performed at admission was normal. Not specific treatment for the virus was required.

A homeless, smoker patient, with active alcohol abuse and medical history of decompensated HCV (treated)-alcohol cirrhosis Child Pugh $\mathrm{C} 10, \mathrm{SBP}$, refractory ascites treated by large-volume paracentesis, was admitted to our gastroenterology unit for encephalopathy grade
2 and an increase waist size. After blood test (Figure 2), urinary test, abdominal-chest CT scans and diagnostic paracentesis, SBP was identified. A multiresistant Escherichia coli was identified in the culture of the ascitic liquid, treatment with piperacillin / tazobactam was started. On day 2 of his admission, all patients in our unit were tested for Sars-Cov2 virus due to a new hospital policy. He presented a positive Sars-Cov2 PCR, and he was transferred to infectious diseases service. He reported a chronic cough, without dyspnea or fever. A new chest radiography showed an increased density in both hemithorax, without clear pneumonia imagen. Hydroxychloroquine was started for 5 days without side effects. After the resolution of the SBP, the patient was discharged.

\section{Discussion}

Patients with cirrhosis are known to have abnormalities of immune function (immunodeficiency), as well as systemic inflammation, which is the pathophysiological hallmark of increased susceptibility to infection2. Patients with cirrhosis are at a high risk of having a severe course of the influenza, including the development of organ failures [3]. However, there is limited data on the course of Covid-19 in patients with chronic liver disease (CLD) and whether SARS-Cov2 can increase the risk of decompensation or development of acute-on-chronic liver failure (ACLF). Current data indicates a prevalence (0-11\%) of pre-existing liver conditions in patients with COVID-19 [4-6]. Is important to note that the exact cause of pre-existing liver conditions has not been outlined.

*Correspondence to: Pilar del Pino Bellido, Gastroenterology Department, University Hospital Virgen Macarena, C/ Dr. Fedriani 3, 41009, Seville, Spain, Tel: +34628486593; E-mail: pilardelpino4@gmail.com

Key words: Chronic liver disease, cirrhosis, COVID-19

Received: August 03, 2020; Accepted: August 10, 2020; Published: August 13, 2020 


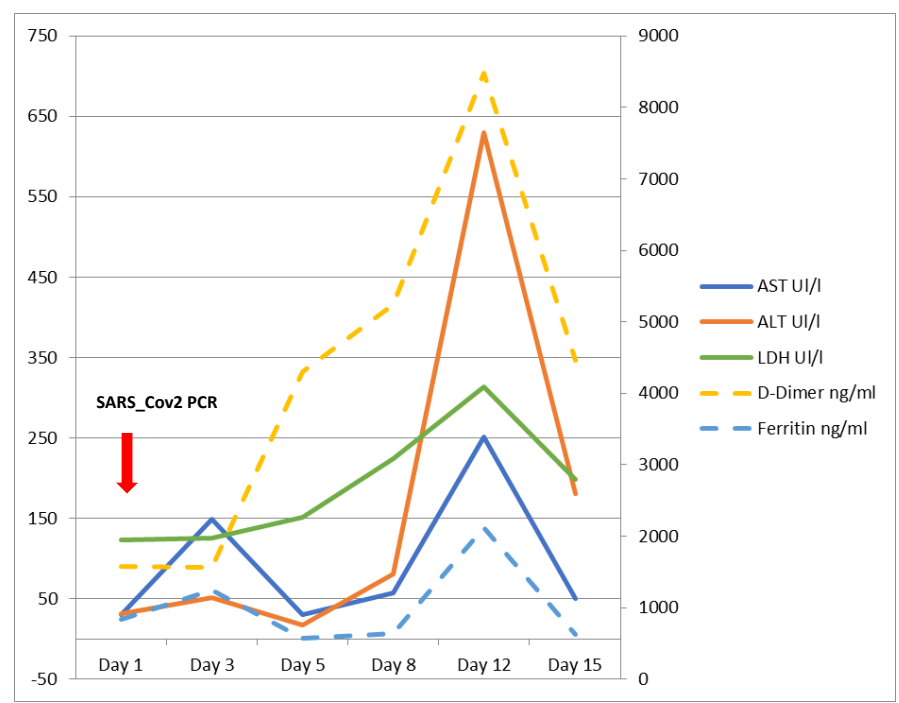

Figure 1. Analytical values during admission in patient 1. AST, ALT, and LDH values are displayed on the left axis, while D-Dimer and ferritin values are displayed on the right axis

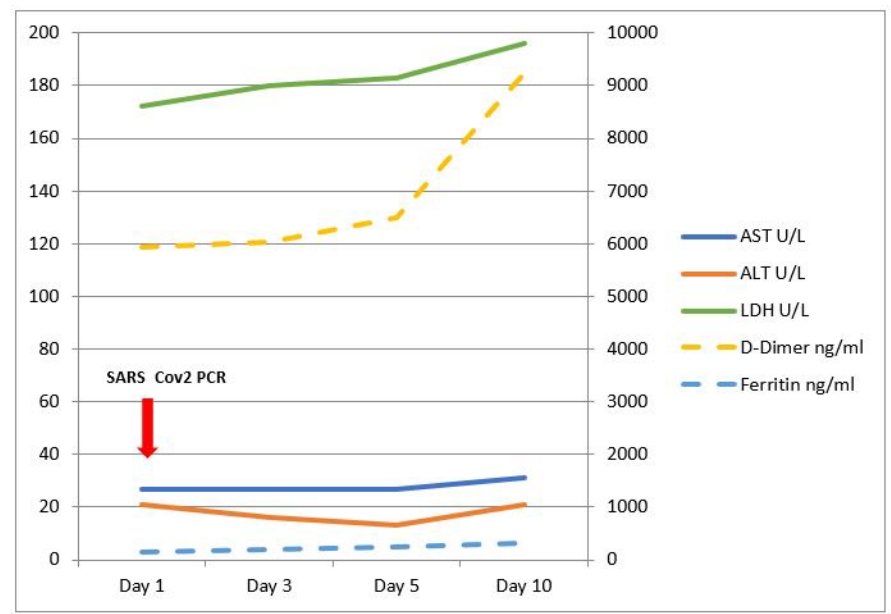

Figure 2. Analytical values during admission in patient 2. AST, ALT, and LDH values are displayed on the left axis, while D-Dimer and ferritin values are displayed on the right axis

This leads us to think about the lower risk of SARS-Cov2 infection in patients with CDL, or alternatively the lack of testing to confirm infection in admitted cirrhotic patients with liver decompensation. Furthermore, immune-mediated inflammatory disease such as rheumatoid arthritis or inflammatory bowel disease are also not mentioned in the published data [6].

Critical host immune factors underlying the development of a severe inflammatory response in some patients with Covid-19 remain poorly defined. Children who experienced mild symptoms or were asymptomatic tend not to develop serious disease despite being able to experience high viral titers. Older people or with comorbidities, meanwhile, are more likely to develop a dysfunctional immune response that fails to eradicate the virus [7].

Cytokine release syndrome, plays a major role in the pathology of COVID-19, increasing interleukin-6 (IL-6), IL-10, IL-2 and IFN-C levels. IL-6 is one of the key cytokines that exacerbates inflammatory responses, which don't only cause pulmonary injury [1]. Meanwhile, cirrhosis has been associated with increase serum expression of the soluble form of the IL- 6 receptor and sgp130, which is a potent inhibitor of IL-6 signaling. This indicates a resistance to IL-6 [2]. It is unknown if this resistance to IL-6 can play a role in the "better" result of the infection in cirrhotic patients by avoiding a severe systemic inflammatory response.

We present two cases of patients with decompensated liver disease that were evaluated for the SARS-Cov2 due to the new hospital policy of testing all patients who require hospital admission. Neither of them presented the typically symptoms of COVID-19 such as fever, fatigue, dry cough, anosmia, headache, or shortness of breath1. Some studies showed that $14 \%$ to $53 \%$ of patients hospitalized with COVID-19 infection present elevated serum liver biochemistries, primarily elevated AST and ALT, and slightly elevated bilirubin [8,9]. However, in patients with advanced liver disease admitted for a liver decompensation, these abnormalities in the liver tests can lead to confusion. We believe in our patients the mild alteration in liver biochemistries was related with the liver decompensations rather than Covid-19 (Figure 1 and 2).

Although liver damage in patients with Covid-19 may be directly caused by active viral replication in hepatic cells though ACE2 receptor $[9,10]$. We believe that covid-19 did not worsen liver function in our patients, and as such did not increase their risk of mortality or develop ACLF during admission. It could be that the absence of airway damage, has produced a mild disease, in which the immunocompromised state of cirrhotic patients did not play a negative role in the severity of the infection.

We consider it is necessary to test for SARS-Cov2 infection in all admitted cirrhotic patients, to avoid nosocomial transmission and to be able to identify the development of severe complications.

\section{Conflict of interest}

The authors declare that they have no conflict of interest.

\section{References}

1. Liu B, Li M, Zhou Z, Guan X, Xiang Y (2020) Can we use interleukin-6 (IL-6) blockade for coronavirus disease 2019 (COVID-19)-induced cytokine release syndrome (CRS)? J Autoimmun 111: 102452. [Crossref]

2. Albillos A, Lario M, Álvarez-Mon M (2014) Cirrhosis-associated immune dysfunction: Distinctive features and clinical relevance. J Hepatol 61: 1385-1396 [Crossref]

3. Schütte A, Ciesek S, Wedemeyer H, Lange CM (2019) Influenza virus infection as precipitating event of acute-on-chronic liver failure. J Hepatol 70: 797-799. [Crossref]

4. Zhang C, Shi L, Wang FS (2020) Liver injury in COVID-19: management and challenges. Lancet Gastroenterol Hepatol 5: 428-430. [Crossref]

5. Mantovani A, Beatrice G, Dalbeni A (2020) Coronavirus disease 2019 and prevalence of chronic liver disease: A meta-analysis. Liver Int 40: 1316-1320. [Crossref]

6. Richardson S, Hirsch JS, Narasimhan M, Crawford JM, McGinn T, et al. (2020) Presenting Characteristics, Comorbidities, and Outcomes Among 5700 Patients Hospitalized With COVID-19 in the New York City Area. JAMA 323: 2052-2059. [Crossref]

7. Tay MZ, Poh CM, Rénia L, MacAry PA, Ng LFP (2020) The trinity of COVID-19: immunity, inflammation and intervention. Nat Rev Immunol 20: 363-374. [Crossref]

8. Bangash MN, Patel J, Parekh D (2020) COVID-19 and the liver: little cause for concern. Lancet Gastroenterol Hepatol 5: 529-530. [Crossref]

9. Li J, Fan JG (2020) Characteristics and Mechanism of Liver Injury in 2019 Coronavirus Disease. J Clin Transl Hepatol 8: 1-5. [Crossref]

10. Sun J, Aghemo A, Forner A, Valenti L (2020) COVID-19 and liver disease. Liver Int 40: 1278-1281 [Crossref]

Copyright: C2020 Pilar DPB. This is an open-access article distributed under the terms of the Creative Commons Attribution License, which permits unrestricted use, distribution, and reproduction in any medium, provided the original author and source are credited. 